\title{
Automatic Simplification of Complex Building Geometry for Whole-building Energy Simulations
}

\author{
Luis Santos ${ }^{1,2}$, Simon Schleicher ${ }^{1}$, Luisa Caldas ${ }^{1,3}$ \\ ${ }^{1}$ UC Berkeley, College of Environmental Design, Department of Architecture, Berkeley, CA, USA \\ ${ }^{2}$ UC Berkeley, Center for the Built Environment, Berkeley, CA, USA \\ ${ }^{3}$ Lawrence Berkeley Laboratory, Berkeley, CA, USA
}

\begin{abstract}
The energy simulation of buildings with complex curved envelopes is still a difficult task. Although an accurate building representation is desirable for energy analysis, a refined mesh representation of (double)curved facades still requires a significant modeling effort for building energy simulation and in most cases the resulting models entail a simulation overhead that is incompatible with design times. This becomes more relevant in fully automated parametric and building energy optimization studies, where a computer program is responsible to instantiate several variations of a building energy model from parametric conceptual building masses. In such settings, it is mandatory that the generated simulation models are valid (i.e., without errors and with accurate building representation) and it is desirable that they are efficient (i.e., fast to run). To answer these challenges, this paper presents a computational tool that automatically simplifies the geometry of complex curved building envelopes for energy simulation. The tool is based on two heuristics - one that post-rationalizes building surfaces and another that samples large energy models and isolate and simulate their most representative parts. We tested our approach in two building masses with complex curved envelopes. The results show that the two heuristics, either combined or applied separately, are able to generate valid and fast building energy models with minimal impact on simulation output.
\end{abstract}

\section{Introduction}

Recently, 3D parametric design tools, that interface with whole Building Energy Simulation (BES) software and optimization algorithms, promise to facilitate Performance-based Design (PBD) workflows at early design stages. However, modeling differences between Computer-Aid Design (CAD) programs, Building Information Modeling (BIM) tools, and BES software hamper a smooth PBD workflow, often requiring redundant and tiresome manual remodeling or reparameterization tasks.

The accurate description and representation of the building in BES is an ill-defined problem: although is an essential requirement in energy analysis it involves a time cost that is incompatible with design times, particularly at early stages (Picco and Marengo, 2015). This problem is particularly important in the case of complex curved and double-curved building envelopes due to the current modeling challenges of representing such surfaces in BES software.

At early design stages, it is common to use simplified versions of complex building geometry to perform faster simulations with manageable outputs, such as in (Delgarm, Sajadi, and Delgarm, 2016). However, this approach entails tedious re-modeling tasks that, inevitably, expands the gap between the initial building model and the correspondent simplified Building Energy Model (BEM). Moreover, the literature on automated BEM inference and simplification either focuses on the thermal zoning of massing models according to ASHRAE guidelines (ASHRAE, 2013) (Dogan, Reinhart, and Michalatos, 2016; Dogan and Reinhart, 2013; Jones et al., 2013) or in sensitivity analysis of simple box-shaped buildings composed by planar surfaces (Picco and Marengo, 2015; Amitrano et al., 2014). In (Picco and Marengo, 2015), the authors assessed the impact of different BEM simplifications including shading elements and thermal zoning in annual energy simulations. Mostly due to the limited formal range of their models, the authors found that strong simplifications in geometry did not produce relevant deviations in the energy simulation outputs. However, Amitrano et al. (2014) showed that, even in simple shoe-box BEM, the inclusion of detailed geometry reduces the uncertainty of energy simulations by 5 to $15 \%$.

Despite these efforts in studying the impact of geometric detail in BEM, the literature that addresses curved or other types of sophisticated building envelope geometry is still limited. Chatzivasileiadi et al. (2018) conducted a sensitivity analysis on the simplification of simple curved building envelopes in BES. The authors found that a significant reduction of geometry complexity has a negligible impact on energy simulation output. This work also shows that simulation accuracy deteriorates rapidly below a critical threshold. Albeit its valuable contribution, the study mostly focuses in simple extrusions of planar curves (Chatzivasileiadi et al., 2018). The statistical approach in measuring the difference of simulation outputs is also very simple and it does not reflect the best practices of BEM assessment and calibration.

Considering that current digital fabrication technology, CAD and BIM tools facilitate the generation and fabrication of buildings with intricate geometries, and that architects are increasingly adopting such geometries in their formal discourse, we argue that it is relevant to 
include complex curved building envelopes in energy simulation studies. Current simplification techniques of complex curved geometry in BEM are mainly $a d h o c$, and their effectiveness heavily depends on the energy modeler experience. Thus, we need better modeling guidelines, approaches, and tools to deal with complex curved building envelopes in BES. Such guidelines and methods would be particularly important for non-expert building energy software users and for automated search procedures used in building energy optimization.

\section{Research goals}

This work targets the current gaps in the literature regarding the automatic simplification of complex curved building geometry for fast and accurate BES. Our study aims to answer two essential research questions:

1) How to automatically simplify early-stage building models, namely those with single- or double-curved envelopes?

\section{2) How far can that simplification go?}

To answer these questions, the authors developed and computationally implemented two envelope geometry simplification heuristics for BEMs. The first is a tessellation procedure that generates a low-poly mesh version of the building's envelope while preserving the original volume. The second proposes a zone sampling technique that reduces a complex multizone BEM to its most representative parts. The result is a novel digital tool that automatically simplifies complex and convoluted building envelopes for BES. The authors tested this tool in two different experiments in order to discuss potential modeling recommendations for mesh reduction in BES, particularly at early design stages.

\section{Methods}

This paper proposes the computational implementation of the two heuristics for automated simplification of complex curved building envelopes in BES. The first heuristic tessellates the building's envelope into a simpler low-polygon mesh, composed by planar faces. By dividing the original the surface width $(\mathrm{u})$ and length (v) the proposed approach generates an approximated mesh formed by quadrilateral faces, i.e., quads. If planar quads do not entirely compose the resulting mesh, the heuristic remeshes the envelope surface using a quasi-isothermic planarization algorithm (Rörig et al., 2015; Sechelmann, Rörig, and Bobenko, 2013) and already used in the context of BES (Santos, Schleicher, and Caldas, 2017). If the planarization step is not successful in planarizing all quads, the heuristic will split the resulting non-planar quads into tri-meshes. The simplification of the building's envelope into a low-poly mesh directly impacts the original volume of air of the thermal zone or zones, thus introducing deviations to the heat balance calculation. To avoid volume deviations, our approach automatically scales the simplified thermal model in order to match the original volume. Equation (1) determines the factor of the uniform 3D scaling $\left(\mathrm{S}_{\text {factor }}\right)$ for the $\mathrm{x}-, \mathrm{y}-$, and $\mathrm{z}$-direction.

$$
S_{\text {factor }}=(\text { original volume } / \text { simplified volume })^{(1 / 3)}(1)
$$

The second proposed approach simplifies multizone BEMs by decomposing them into a set of representative single zone models. The user can manually identify and isolate the sample thermal zones by drawing a polygon over the model or let the system automatically find and isolate the representative thermal parts. Currently, the implemented heuristic uses information regarding orientation, zone program, schedules, loads, and other zone properties such as heating and cooling set points to perform this automatic subdivision. After subdividing the BEM into representative single thermal zones $(\mathrm{Zi})$, the system calculates the original volume of influence of each representative zone (Vzi) and closes the sampled simplified thermal model with adiabatic surfaces. Then, the tool simulates each single thermal zone in parallel and normalizes the simulation outputs in a volume-based Energy Use Intensity (EUIv), as an alternative to the typical EUI that normalizes energy by unit area. Equation (2) is then used to find the energy consumption of any energy end-use of the entire building, which sums the energy consumption of all representative zones $\mathrm{Zi}$, which in turn result from the multiplication of the correspondent EUIv by Vzi.

$$
\text { Energy consumption }(Z i)=\sum_{i=1}^{n}\left(V_{z i} \times \operatorname{EUIv}(z i)\right)(2)
$$

This work presents two experiments that assess the performance of the proposed approaches in automatically simplifying BEM in terms of run time and energy simulation output deviation. In both experiment, we compare the simplified BEM (i.e., a BEM with a coarser geometric resolution) results with the ones from a BEM with a high geometric resolution using the following metrics: simulation time, measured in seconds, the Coefficient of Variation of the Root Mean Squared Error (CVRMSE), and Normalized Mean Bias Error (NMBE). In this case, NMBE provides the average of the errors of the BEMs with simplified versions of the building envelope (the simplified models) normalized by the mean of the results of a BEM with a highly detailed envelope representation, the benchmark model. In NMBE, negative values mean over-prediction while positive values indicate underprediction. Because NMBE is susceptible to the cancelation effect, it needs to be complemented with CVRMSE, a statistical index that is not affected by it. In our experiments, CVRMSE measures the variability of the errors between the benchmark BEM and the different simplified BEMs. NMBE is presented in equation (3) while CVRMSE in (4). In both equations $d_{i}$ is the simulated data of the high-resolution model at hour $i, s_{i}$ is the predicted value of the simplified BEM, $n$ is the number of hours $i$ simulated, $\bar{d}$ is the mean of the detailed model data.

$$
\begin{gathered}
N M B E=1 / \bar{d} \times \sum_{i=1}{ }^{n}(d i-s i) / n \times 100(\%) \\
C V R M S E=1 / \bar{d} \times \sqrt{\sum_{i=1}{ }^{n}\left(d_{i}-s_{i}\right)^{2} /(n-1) \times 100(\%)}
\end{gathered}
$$

Table 1 shows ASHRAE Guideline 14 (ASHRAE, 2002) acceptability thresholds for CVRMSE and NMBE for hourly building energy simulations. Although, these guidelines directly apply in BES calibration studies (i.e., compare BEM results with measured data), this work uses them to study the impact of different geometric 
resolutions of complex (double)curved building envelopes in simulation outputs. We assume that the higher the geometric resolution of a BEM the higher the accuracy of the simulation results. Thus, the benchmark case used to compare different levels of resolution is the BEM with the highest resolution produced by our approach.

Table 1: ASHRAE Guideline 14 for calibration criteria.

\begin{tabular}{|c|c|c|}
\hline Data type & NMBE (\%) & CVRMSE (\%) \\
\hline Hourly & \pm 10 & 30 \\
\hline
\end{tabular}

The authors used EnergyPlus (Crawley et al., 2001) to conduct all whole building energy simulations and Rhino 3D modeling CAD software to generate the initial geometry of the models. Grasshopper, a visual programming language for Rhino, and Python allowed the computational implementation of the BEM simplification heuristics proposed in this paper.

All simulations used Typical Meteorological Year (TMY) data from Oakland's Airport. Table 2 presents the thermal and optical properties of the building envelope used in both experiments. The thermal properties of the opaque surfaces align with ASHRAE 90.1 standard (ASHRAE, 2013) recommendations for Oakland's ASHRAE climate zone (3). Since ASHRAE 90.1 recommended glazing for climate zone 3 has a low visible transmittance (VT), the thermal and optical properties used in this work is of a clear, low-emissivity double-glazed unit that balances well thermal and daylight performance.

Table 2: Building envelope thermal and optical properties used in the experiments

\begin{tabular}{|l|l|}
\hline \multicolumn{2}{|c|}{ Opaque Surfaces } \\
\hline Building Element & R-value (m2·K/W) \\
\hline Floor & 1.8 \\
\hline Walls & 1.95 \\
\hline Roof Glazing Surfaces \\
\hline \multicolumn{2}{|c|}{1.95} \\
\hline U value $\left(\mathrm{W} / \mathrm{m}^{2} \cdot \mathrm{K}\right)$ & 2.56 \\
\hline $\begin{array}{l}\text { Solar Heat Gain Coefficient } \\
\text { (SHGC) }\end{array}$ & 0.27 \\
\hline Visible Transmittance (VT) & 0.64 \\
\hline
\end{tabular}

Table 3 lists the main simulation parameters used in the EnergyPlus simulations including internal loads, HVAC system properties, thermostat, infiltration and ventilation rates, and daylighting controls.

Table 3: EnergyPlus simulation parameters

\begin{tabular}{|l|l|}
\hline \multicolumn{2}{|l|}{ Internal Loads } \\
\hline People (ppl) & $\begin{array}{l}0.05 \mathrm{ppl} / \mathrm{m}^{2} \text {, with a typical office } \\
\text { schedule }\end{array}$ \\
\hline Lights & $\begin{array}{l}11.8 \mathrm{~W} / \mathrm{m}^{2} \text {, with a typical office } \\
\text { schedule and daylight dimming }\end{array}$ \\
\hline Equipment & $\begin{array}{l}7.64 \mathrm{~W} / \mathrm{m}^{2}, \text { with a typical office } \\
\text { schedule }\end{array}$ \\
\hline & HVAC System \\
\hline Type & EnergyPlus Ideal Load Air System \\
\hline Cooling COP* & 3.5 \\
\hline Heating COP* & 0.9 \\
\hline Economizer & None \\
\hline Outdoor Air & $0.14 \mathrm{cfm}$ per person \\
\hline
\end{tabular}

\begin{tabular}{|l|l|}
\hline \multicolumn{2}{|c|}{ Thermostat } \\
\hline Cooling setpoint & $26^{\circ} \mathrm{C}$, with a typical office schedule \\
\hline Cooling setback & $32^{\circ} \mathrm{C}$ \\
\hline Heating setpoint & $21^{\circ} \mathrm{C}$ \\
\hline Heating setback & $18^{\circ} \mathrm{C}$ \\
\hline \multicolumn{2}{|c|}{ Daylight Controls } \\
\hline Type & $\begin{array}{l}\text { Continuous off dimming control } \\
\text { with typical office schedule }\end{array}$ \\
\hline $\begin{array}{l}\text { Minimum Input } \\
\text { Power Fraction }\end{array}$ & 0.1 \\
\hline $\begin{array}{l}\text { Minimum Light } \\
\text { Output Fraction }\end{array}$ & 0.1 \\
\hline Illuminance Setpoint & 300 lux \\
\hline $\begin{array}{l}\text { Zone Fraction per } \\
\text { daylight sensor }\end{array}$ & $1 /$ Number of daylight sensors \\
\hline * Coefficient of Performance \\
\hline
\end{tabular}

The simulations report annual data on an hourly basis for heating, cooling, lighting, and process loads. The following discuss in more detail each experiment.

\section{Experiment 1}

This experiment assess the impact of simplifying complex double-curved building envelopes in BES outputs. The authors seek to find how to rationalize the geometry of complex curved building surfaces for BES. We particularly seek the optimal degree of geometry simplification in terms of simulation time and accuracy. In this experiment, the authors used the massing model illustrated in Figure 1. The model is a $1700 \mathrm{~m}^{3}$ doublecurved pavilion with a varying depth $(7.5 \mathrm{~m}$ to $16.8 \mathrm{~m})$, $25 \mathrm{~m}$ of length, and height $(3.75 \mathrm{~m}$ to $8.4 \mathrm{~m})$ inspired in the Landesgartenschau Exhibition Hall pavilion in Stuttgart, Germany (Krieg et al., 2015) and in the Sage Gateshead building designed by Norman Foster architects.

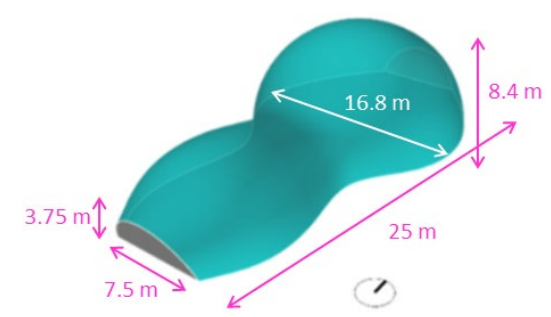

Figure 1: Initial massing model composed of Non-

Uniform Rational Basis Spline (NURBS) surfaces.

Using the first geometry simplification heuristic, our tool generated 10 different BEM from the initial massing model with varying mesh resolutions, i.e., quad count. All BEM have a Window-to-Wall-Ratio (WWR) of 40\%. Initially, the authors used the NURBS surface parser available in Honeybee (Roudsari, Pak, and Smith, 2013), a popular EnergyPlus and Radiance front-end for Grasshopper, to produce the benchmark energy model, i.e. the high polygon count BEM used to assess the impact of geometry reduction/simplification in simulation output. The Honeybee resulting BEM (Figure 2) encompassed 6,278 faces, and it was unable to run in useful time - after 4 hours the energy simulation was still initializing warmup calculations. Thus, the BEM consider as the comparison benchmark in this experiment was the 
model generated by our approach with a high mesh polygon count.

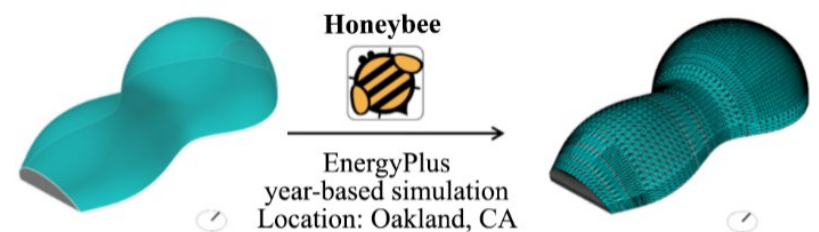

Conceptual massing model

Honeybee model: 6,278 faces

Figure 2: Initial massing model (left) and resulting

Honeybee BEM. Due to the high polygon mesh count, the BEM was unable to run in EnergyPlus in useful time.

Table 4 lists the different simplified models along with the original envelope surface $u$ and $v$ subdivisions and the resulting main and subsurfaces (windows and skylights) count. A thicker box marks the benchmark BEM.

Table 4: BEMs generated by the proposed approach for the initial massing model.

\begin{tabular}{|c|c|c|c|}
\hline $\begin{array}{c}\text { BEM } \\
\text { ID }\end{array}$ & $\begin{array}{c}\text { Envelope } \\
\text { subdivisions }(u, v)\end{array}$ & $\begin{array}{c}\text { Main } \\
\text { Surfaces }\end{array}$ & Subsurfaces \\
\hline Res01 & $(4,4)$ & 26 & 16 \\
\hline Res02 & $(6,6)$ & 52 & 36 \\
\hline Res03 & $(8,8)$ & 86 & 64 \\
\hline Res04 & $(10,10)$ & 126 & 100 \\
\hline Res05 & $(12,12)$ & 178 & 144 \\
\hline Res06 & $(14,14)$ & 236 & 196 \\
\hline Res07 & $(16,16)$ & 298 & 256 \\
\hline Res08 & $(18,18)$ & 376 & 324 \\
\hline Res09 & $(20,20)$ & 457 & 400 \\
\hline Res10 & $(22,22)$ & 543 & 484 \\
\hline
\end{tabular}

Although our approach ensures that the resulting simplified BEM maintains the air volume of the benchmark model, it is important to measure the deviations on external surface area, since the heat transfer through the envelope is directly correlated to it. Table 5 compares relevant envelope surface parameters including form-factor (i.e., surface-to-volume ratio) and area of surface. The approach keeps the deviation very low both in envelope surface area and in form factor, thus minimizing any simulation output errors due to differences in surface area.

Table 5: BEMs form parameters comparison.

\begin{tabular}{|c|c|c|c|c|}
\hline $\begin{array}{c}\text { BEM } \\
\text { ID }\end{array}$ & $\begin{array}{c}\text { Surface } \\
\text { Area (Sa) } \\
{\left[\mathbf{m}^{2}\right]}\end{array}$ & $\begin{array}{c}\text { Sa\% error } \\
\text { (compared } \\
\text { with Res10) }\end{array}$ & $\begin{array}{c}\text { Form } \\
\text { Factor } \\
\text { (FF) }\end{array}$ & $\begin{array}{c}\text { FF\% error } \\
\text { (compared } \\
\text { with Res10) }\end{array}$ \\
\hline Res01 & 1005.3 & $5 \%$ & 0.592 & $5 \%$ \\
\hline Res02 & 979.1 & $2 \%$ & 0.576 & $2 \%$ \\
\hline Res03 & 970.5 & $1 \%$ & 0.571 & $1 \%$ \\
\hline Res04 & 966.4 & $1 \%$ & 0.568 & $1 \%$ \\
\hline Res05 & 964.3 & $0 \%$ & 0.567 & $0 \%$ \\
\hline Res06 & 962.9 & $0 \%$ & 0.567 & $0 \%$ \\
\hline Res07 & 961.9 & $0 \%$ & 0.566 & $0 \%$ \\
\hline Res08 & 961.3 & $0 \%$ & 0.566 & $0 \%$ \\
\hline Res09 & 960.9 & $0 \%$ & 0.565 & $0 \%$ \\
\hline Res10 & 960.5 & N.A. & 0.565 & N.A. \\
\hline
\end{tabular}

Table 6 assesses the difference between glazed area consider as window and as skylight. This is a sensitive modeling point since there are differences in terms of the heat transfer coefficients applied to different tilts of glazed surfaces. Our approach automatically dispatches envelope surfaces as roofs or walls, and consequently as windows or skylights, depending on the tilt angle of the surface. Walls and windows are assigned to surfaces with a tilt angle between $\geq 60^{\circ}$ and $\leq 120^{\circ}$. The deviation variability presented in Table 6 shows is a direct application of this rule. Nevertheless, starting from Res03, the deviation error is acceptable for the area assigned to skylights and windows in the simplified models $(\leq|10| \%)$.

Table 6: BEMs' window and skylight area comparison.

\begin{tabular}{|c|c|c|c|c}
\hline $\begin{array}{c}\text { BEM } \\
\text { ID }\end{array}$ & $\begin{array}{c}\text { Window } \\
\text { Area } \\
(\text { Warea) } \\
{\left[\mathbf{m}^{2}\right]}\end{array}$ & $\begin{array}{c}\text { Warea \% } \\
\text { error } \\
(\text { compared } \\
\text { with Res10) }\end{array}$ & $\begin{array}{c}\text { Skylight } \\
\text { Area } \\
\text { (Sk_area) } \\
{\left[\mathrm{m}^{2}\right]}\end{array}$ & $\begin{array}{c}\text { Sk_area } \\
\text { \% error } \\
(\text { compared } \\
\text { with Res10) }\end{array}$ \\
\hline Res01 & 149.8 & $-21 \%$ & 87.64 & $105 \%$ \\
\hline Res02 & 176.61 & $-7 \%$ & 57.52 & $35 \%$ \\
\hline Res03 & 194.62 & $3 \%$ & 38.59 & $-10 \%$ \\
\hline Res04 & 188.7 & $0 \%$ & 44.08 & $3 \%$ \\
\hline Res05 & 187.8 & $-1 \%$ & 44.74 & $5 \%$ \\
\hline Res06 & 191.46 & $1 \%$ & 40.9 & $-4 \%$ \\
\hline Res07 & 185.44 & $-2 \%$ & 46.8 & $9 \%$ \\
\hline Res08 & 191.2 & $1 \%$ & 40.95 & $-4 \%$ \\
\hline Res09 & 192.77 & $2 \%$ & 39.33 & $-8 \%$ \\
\hline Res10 & 189.31 & N.A. & 42.75 & N.A. \\
\hline
\end{tabular}

All the energy models have three daylight sensors equally spaced located along the central North-South axis at $0.8 \mathrm{~m}$ height. Figure 3 shows three of the generated BEMs, each one illustrating different levels of envelope mesh resolution; low (Res01), intermediate (Res04), and high (Res10).

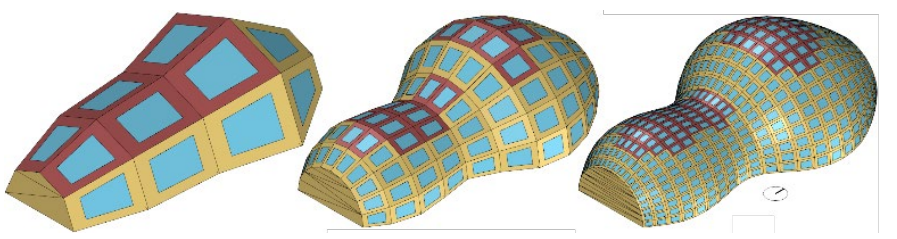

Figure 3: Different levels of envelope geometric resolution. From left to right: Res01, Res04, and Res 10, the BEM with higher polygon count and considered as the benchmark model in this experiment.

This experiment compared the simulation time, NMBE and CVRMSE for heating, cooling, lighting, process, and total energy of the benchmark BEM (Res10) against the other coarser energy models.

\section{Experiment 2}

This experiment assesses the performance of the second simplification heuristic that decomposes a multizone model into simpler representative single thermal zone models. The aim is to estimate the overall results of a larger BEM by quickly simulating smaller parts of it, thus saving simulation time and reducing the effort in complex modeling tasks. To test this heuristic, the authors used a torus building mass with a volume of approximately 
$27,426 \mathrm{~m}^{3}$, an inner radius of $12 \mathrm{~m}$, an outer radius of 24 $\mathrm{m}$, and an arched section with $12 \mathrm{~m}$ high. This massing model is inspired on funnel structures of contemporary large commercial buildings such as the ones of the Milano Trade Fair, design by Massimiliano and Doriana Fuksas, or the ones proposed by Norman Foster Architects in the scheme for the new Mexico building airport. Figure 4 shows how our tool automatically processed the curved building envelope of the initial torus mass into two BEM with a WWR of $60 \%$; one with a high and another with a coarser geometric resolution. Since this heuristic is based on slicing a larger BEM model into single zone models that would be capped on the sides it is important that the different thermal zones are physically separated by a partition wall. Thus, zones are separated by a typical low mass partition walls with an R-value of $0.39 \mathrm{k} . \mathrm{m}^{2} / \mathrm{W}$.

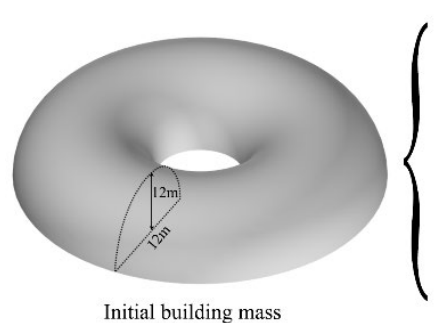

Initial building mass

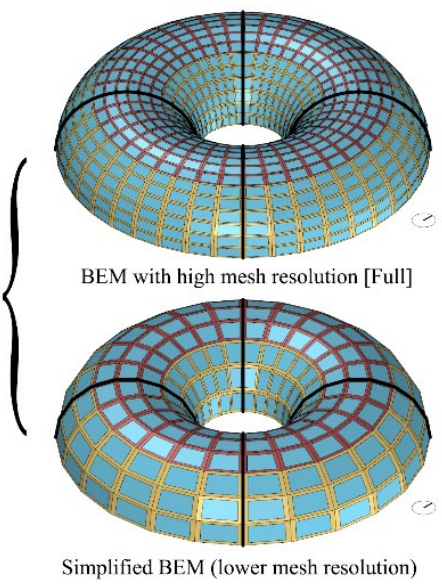

Figure 4: Original torus mass building mass (left) and the correspondent generated BEM with different mesh resolutions (right). The thicker black lines represent thermal zone division.

After generating the BEM, our approach isolates a representative part of each thermal zone. Figure 5 shows the sliced representative zones generated by our tool for the high-resolution BEM and its simplified counterpart. Each slice is caped on the sides with adiabatic surfaces, colored in magenta.
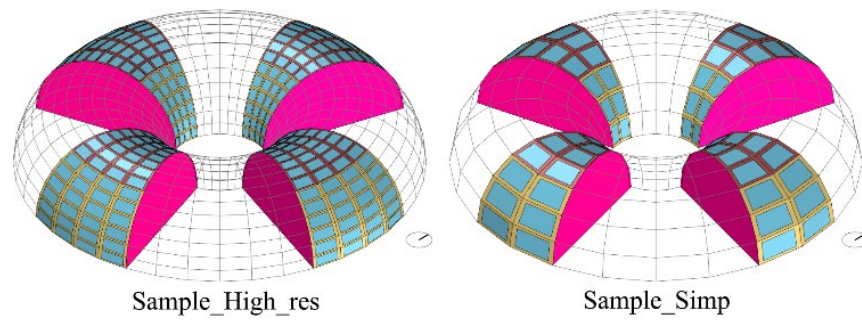

Figure 5: Representative zones used in Experiment 2.

Left: BEM with a high-resolution envelope

(Sample_High_res). Right: BEM with simplified geometry (Sample_Simp).

Table 7 compares the geometry of the different BEM used in this experiment. Full_High_res is the detailed 4 zone energy model, the benchmark BEM in this experience. Sample_High_res corresponds to the four resulting single thermal zone models produced by simply slicing Full_High_res. Sample_Simp models result from using the first simplification heuristic to simplify the envelope of Full_High_res and then subsequently slicing it.

Table 7: BEMs generated by the proposed approach for the initial massing model.

\begin{tabular}{|c|c|c|}
\hline BEM ID & $\begin{array}{c}\text { Number of } \\
\text { Main Surfaces }\end{array}$ & $\begin{array}{c}\text { Number of Sub } \\
\text { Surfaces (windows) }\end{array}$ \\
\hline Full_High_res & 732 & 480 \\
\hline Sample_High_res & $\begin{array}{l}588 \text { (147 per } \\
\text { single zone) }\end{array}$ & $\begin{array}{c}288 \text { (72 per single } \\
\text { zone) }\end{array}$ \\
\hline Sample_Simp & $\begin{array}{l}156 \text { (39 per } \\
\text { single zone) }\end{array}$ & $\begin{array}{c}72 \text { (18 per single } \\
\text { zone) }\end{array}$ \\
\hline
\end{tabular}

This experiment compared the results of Sample_High_res and Sample_Simp against the outputs of the benchmark BEM, Full_High_res.

\section{Results}

\section{Experiment 1}

To find a relation between geometric resolution and simulation time, for each BEM produced by our approach, the authors paired run time with the number of main surfaces. Figure 6 shows a plot of those pairs with a thirdorder polynomial trendline fit to the points. The coefficient of determination of the polynomial fit $\left(\mathrm{R}^{2}\right)$ is 0.98 . Using this regression model an user can reasonably predict the amount of simulation time that a certain degree of simplification entails in this specific case.

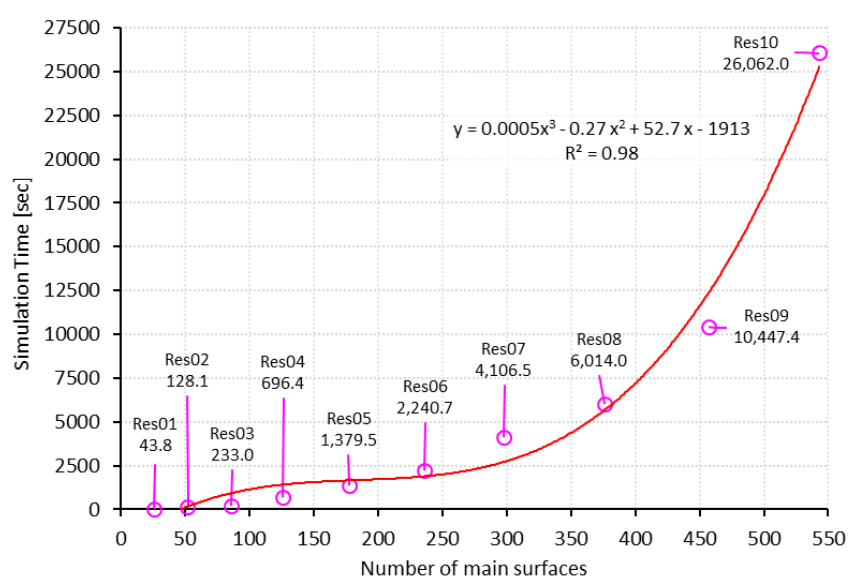

Figure 6: Relation between the number of main surfaces and simulation time. Each data point is a BEM generated by the proposed approach.

Figures 7 and 8 show the relation between the geometric complexity of each simplified BEM and CVRMSE NMBE. CVRMSE and NMBE use Res10 as the benchmark BEM and are break down by energy end-use; cooling, heating, lighting, process, and total energy.

Finally, Table 8 relates the percentage of geometry reduction, simulation speed, and CVRMSE and NMBE of the total estimated building energy consumption for each simplified BEM relatively to the benchmark model (Res10). 


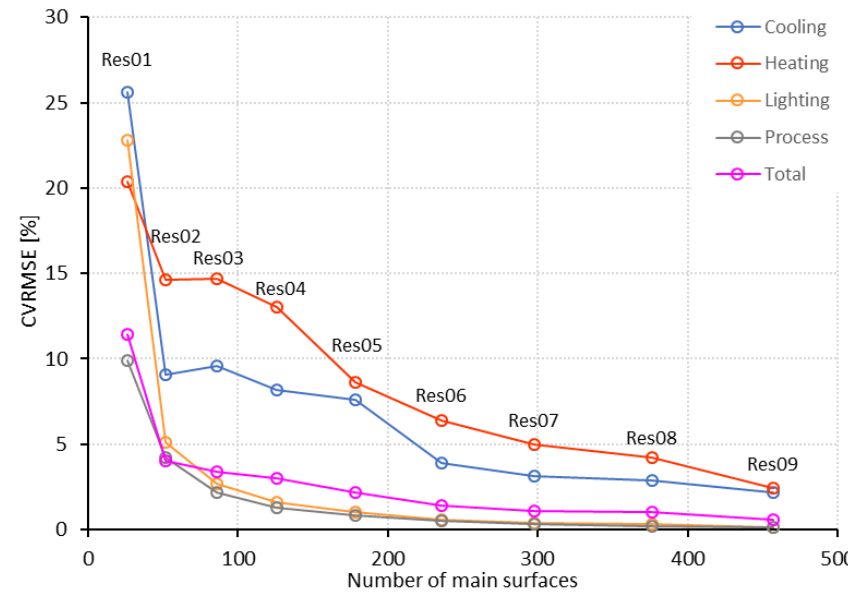

Figure 7: Geometry complexity and CVRMSE of each simplified BEM per energy end-use.

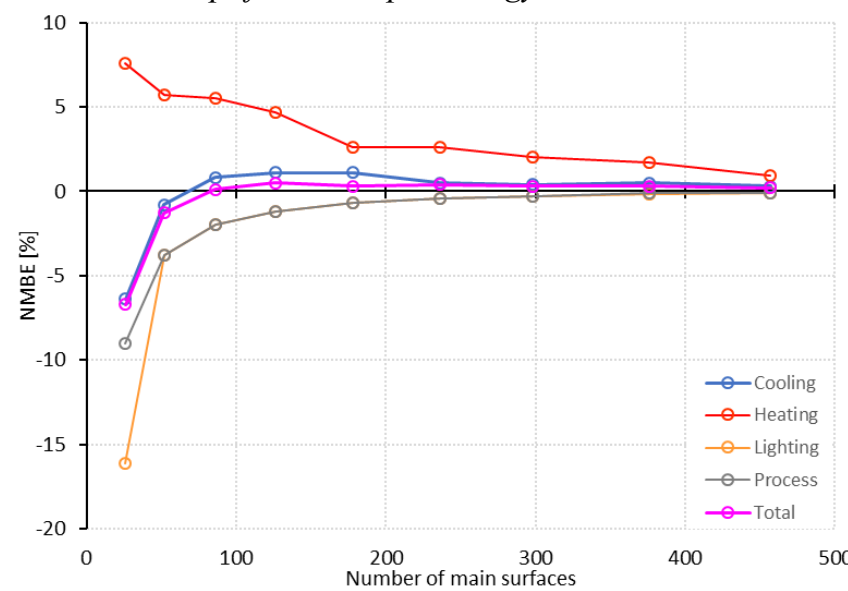

Figure 8: Geometry complexity and NMBE of each simplified BEM per energy end-use.

Table 8: Geometry reduction, relative run speed, and CVRMSE and NMBE of each simplified BEM.

\begin{tabular}{|c|c|c|c|c|}
\hline $\begin{array}{c}\text { BEM } \\
\text { ID }\end{array}$ & $\begin{array}{c}\text { Geometry } \\
\text { reduction }\end{array}$ & $\begin{array}{c}\text { CVRMSE } \\
\text { (total energy) }\end{array}$ & $\begin{array}{c}\text { NMBE } \\
\text { (total energy) }\end{array}$ & $\begin{array}{c}\text { x faster } \\
\text { than Res10 }\end{array}$ \\
\hline Res01 & $95.2 \%$ & $11.4 \%$ & $-6.7 \%$ & 595 \\
\hline Res02 & $90.4 \%$ & $4 \%$ & $-1.3 \%$ & 204 \\
\hline Res03 & $84.2 \%$ & $3.4 \%$ & $0.1 \%$ & 112 \\
\hline Res04 & $76.8 \%$ & $3 \%$ & $0.5 \%$ & 37 \\
\hline Res05 & $67.2 \%$ & $2.2 \%$ & $0.3 \%$ & 19 \\
\hline Res06 & $56.5 \%$ & $1.4 \%$ & $0.4 \%$ & 12 \\
\hline Res07 & $45.1 \%$ & $1.1 \%$ & $0.3 \%$ & 6 \\
\hline Res08 & $30.8 \%$ & $1 \%$ & $0.3 \%$ & 4 \\
\hline $\operatorname{Res} 09$ & $15.8 \%$ & $0.6 \%$ & $0.2 \%$ & 2.5 \\
\hline
\end{tabular}

\section{Experiment 2}

Tables 9, 10, and Figure 9 present the results of this experiment, capturing the two tests performed: (1) sampling, isolating, and simulating the detailed multizone BEM (Full_High_res) in four detailed samples (Sample_High_res), (2) further simplify the geometry of the samples (Sample_Simp) by adopting the heuristic used in Experiment 1. Table 9 shows the simulation time of the Full_High res, Sample High res, and Sample_Simp. It also lists the \% of simulation time reduction of the simplified BEMs in comparison with Full_High_res.

Table 9: Simulation time of the three BEM approaches and reduction of simulation time of the simplified BEMs.

\begin{tabular}{|l|c|c|}
\hline \multicolumn{1}{|c|}{ BEM ID } & $\begin{array}{c}\text { Simulation time } \\
\text { (seconds) }\end{array}$ & $\begin{array}{c}\text { \% of simulation } \\
\text { time reduction }\end{array}$ \\
\hline Full_High_res & 15,407 & N.A. \\
\hline Sample_High_res & 644,4 & 95.8 \\
\hline Sample_Simp & 89 & 99.4 \\
\hline
\end{tabular}

Table 10: Geometry reduction of the simplified BEM and correspondent CVRMSE, NMBE, and improvement of simulation run time.

\begin{tabular}{|l|c|c|c|c|}
\hline BEM ID & $\begin{array}{c}\text { Geometry } \\
\text { reduction }\end{array}$ & $\begin{array}{c}\text { CVRMSE } \\
\text { (total } \\
\text { energy) }\end{array}$ & $\begin{array}{c}\text { NMBE } \\
\text { (total } \\
\text { energy) }\end{array}$ & $\begin{array}{c}\text { x faster than } \\
\text { Full_High_res }\end{array}$ \\
\hline $\begin{array}{l}\text { Sample_ } \\
\text { High_res }\end{array}$ & $20 \%$ & $6.4 \%$ & $-1.4 \%$ & 24 \\
\hline $\begin{array}{l}\text { Sample_ } \\
\text { Simp }\end{array}$ & $79 \%$ & $7.2 \%$ & $-1.7 \%$ & 173 \\
\hline
\end{tabular}

Finally, Figure 9 presents a full breakdown of the error metrics CVRMSE and NMBE by energy end-use for the two simplified BEMs.

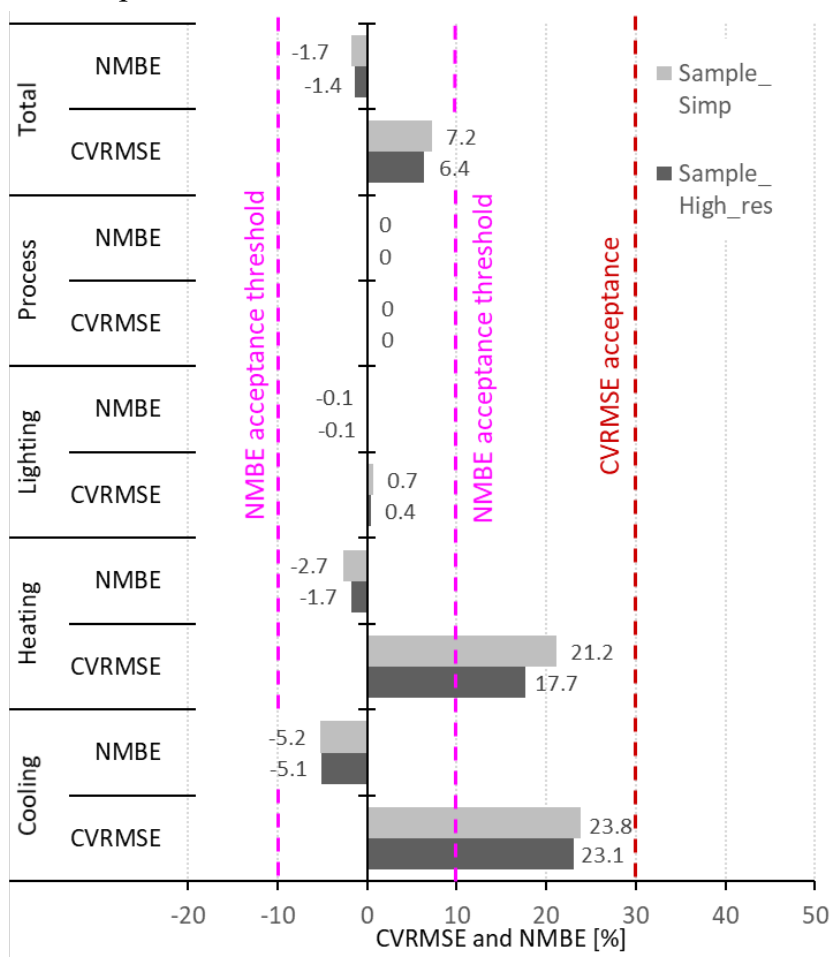

Figure 9: CVRMSE and NMBE of the simplified BEMS for each energy end-use.

\section{Discussion}

Both experiments show that a direct translation of complex double curved building geometry to a BEM entails considerable computational overheads, making such literal translations useless and undesirable for design workflows based on building energy performance. The results show that automatic simplifications are useful in delivering faster and efficient simulation models as long as they capture the overall building shape, maintain the 
same zone air volume, and keep deviations to the external surface area minimal.

In Experiment 1, we observe an exponential relationship between simulation run time and geometric complexity, measured in terms of the number of quad panels necessary to describe a complex curved envelope in BES. Figure 6 clearly shows that small increments in surface subdivision yield a significant impact in simulation time. However, Figure 7 and 8 demonstrate that high levels of simplification are extremely useful in reducing the simulation time at a minimal cost in simulation output results. They also show that extreme simplifications CVRMSE and NMBE rapidly increase. Albeit the simpler BEM, Res01, presents acceptable CVRMSE and NMBE for most of the energy end uses, we observe a drastic spike in this point. The error is more evident for lighting and heating, indicating that high levels of geometric simplification might fail in fully capturing solar-related phenomena such as solar heat gains and daylighting. In fact, Res01' NMBE in lighting is not acceptable ( $>|10| \%)$. It is plausible to assume that if we increase the level of simplification, for example to only $2 \mathrm{u}$ and $\mathrm{v}$ subdivisions of the shell surface, that both heating and lighting would also not fulfill the acceptance criteria for both error metrics. This indicates that reducing envelope dominated buildings with complex curved facades to shoe box BEM results in an oversimplification that will likely fail in properly model the heat transfer phenomena. Considering Figure 6 and 7 and the results listed in Table 8 , we recommend a maximum of $\approx 80 \%$ of reduction of the original number of polygons that describe complex envelopes' geometry. Res03 $(\approx 84 \%$ of geometry reduction) shows an excellent balance between error and simulation time - it ran in $3 \mathrm{~min}$ and 53 seconds (112 times faster than Res10) and shows minimal error in total energy consumption; CVRMSE and NMBE is $3.4 \%$ and $0.1 \%$ respectively.

The heuristic studied in Experiment 2 also presents promising results in significantly improving simulation run time with little impact on simulation results. Sampling a multizone model and decomposing it into smaller and simpler single thermal zone models was able to simulate a full detailed, complex multizone BEM in useful time. The combination of this approach with the building envelope geometry simplification used in Experiment 1 yield significant improvements in simulation time with a minimal cost in accuracy. Sample_Simp, the BEM that combines both the thermal zone sampling and surface rationalization approaches, is 7 times faster than Sample_High_res, the BEM that only uses the sampling and decomposition approach. This improvement in run time as a small impact in NMBE (-0.3\%) and CVRMSE $(+0.8 \%)$. Compared with the benchmark model of this experiment, Sample_Simp is 173 faster to run (1 min and 29 seconds) and reports acceptable CVRMSE and NMBE in terms of total energy; $7.2 \%(22.8 \%$ below the acceptance threshold $)$ and $-1.7 \% \quad(8.3 \%$ above the negative range of acceptance threshold) respectively.

Moreover, Figure 9 shows that both Sample_High_res and Sample_Simp solutions energy end-uses are within the acceptance criteria for CVRMSE and NMBE in all energy end uses. As expected, the process loads don't show any deviation since they are exclusively dependent on surface area and schedule and both simplifications introduced minimal deviations to surface area. The error in both Cooling and Heating are similar, indicating that the simplifications can properly capture the overall performance of the highly detailed BEM.

The small difference between Sample High res and Sample_Simp in all energy end-uses also indicates that the combination of the two simplification heuristics is feasible, valid, and beneficial to use in the study of multizone BEMs with complex curved envelopes, particularly at early design stages where faster whole building energy simulations are desirable.

\section{Conclusion}

This work demonstrates that whole building energy simulations are sensitive to geometry complexity, particularly in simulation run time. It argues that a direct translation of complex curved building envelopes is undesirable since it leads to slow simulations that are incompatible with design times, particularly at early stages. To overcome this problem, and to avoid timeconsuming manual remodeling tasks, the authors propose two computational approaches that, from any curved enclosed building envelope, automatically generates simpler and computationally efficient BEM. The first method simplifies complex surfaces that compose the building envelope by producing a low polygon mesh of the original surfaces. The second approach samples a multizone BEM to isolate smaller and representative parts of the original model.

The results presented in this paper demonstrate that both approaches deliver faster BEMs with minimal deviation on simulation outputs. Since EnergyPlus usually assumes a well-mixed air volume for each thermal zone and that the HVAC system used in this work is an ideal and simple air system, it is paramount to preserve the same air volume in simplification processes. Adding to those simulation assumptions the fact that this work focusses in BES at early design phases, the work did not consider thermal stratification in the zones. Future work will contemplate if the proposed simplification processes are useful in other types of HVAC systems, particularly in underfloor air distribution and non-air systems such as radiant floors or ceilings. Because thermal stratification is relevant in such systems, the authors will also investigate the impact of geometric simplification on it.

This work also indicates that extremely simplified models that are unable to capture the overall building shape are likely to be bad surrogates. Future research endeavors will look at deeper plan buildings, which are core dominated, to determine to what extent it is relevant to capture, even in a coarse way, the complex geometry of the envelope.

Both Experiment 1 and 2 shows that the tool proposed in this paper is able to generate simplified BEMs within the acceptable range for CVRMSE and NMBE error metrics. Experiment 2 also shows that is also possible and viable to combine both simplification approaches - geometry 
simplification of building envelopes and the decomposition of a complex BEM into isolated representative surrogates.

Because the experiments only study the impact of geometry simplification in energy simulation results, the authors plan to assess the effectiveness of the tool in generating simplified BEM geometries for calibrated building energy simulations where measured data is available.

Finally, both experiments used TMY data from Oakland, CA. The mild Oakland climate might be smoothing errors; thus, the authors of this paper intend to test the proposed approaches in locations representative of more extreme climates such as Phoenix, AZ (hot and dry), and Boston, MA, (cold winter and hot and humid summers).

In sum, the proposed tool facilitates the effective description of building with complex geometries in the context of BES, particularly for non-expert energy modelers, and yields a high potential to be used in building energy parametric and optimization studies, particularly the ones that use metaheuristics, since the tool can automatically generate valid BEMs that run faster.

\section{Acknowledgment}

Portuguese national funds supported this work through Fundação para a Ciência e a Tecnologia (FCT) with reference SFRH/BD/98658/2013.

\section{References}

Amitrano, L., N. Isaacs, K. Saville-Smith, M. Donn, M. Camilleri, A. Pollard, M. Babylon, R. Bishop, J. Roberti, and L. Burrough (2014). Building Energy End-Use Study (BEES) Part 1: Final Report. BRANZ Study Report SR297/1, BRANZ Ltd, Judgeford, New Zealand.

ASHRAE (2002). Guideline 14-2002, Measurement of Energy and Demand Savings. American Society of Heating, Ventilating, and Air Conditioning Engineers, Atlanta, Georgia.

ASHRAE (2013). ASHRAE Standard 90.1-2013 Determination of Energy Savings: Quantitative Analysis.

Chatzivasileiadi, A., Lila, A., Lannon, S., \& Jabi, W. (2018). The Effect of Reducing Geometry Complexity on Energy Simulation Results. Proceedings from eCAADe 2018: Education and research in Computer Aided Architectural Design in Europe Conference (2), 559-568. Lodz, Poland, 19-21 September 2018.

Crawley, B., L. K. Lawrie, F. Winkelmann, W. Buhl, Y. Huang, C. Pedersen, R. Strand, et al. (2001). EnergyPlus: Creating a New-Generation Building Energy Simulation Program. Energy and Buildings 33(4), 319-331.

Delgarm, N., B. Sajadi, and S. Delgarm (2016). MultiObjective Optimization of Building Energy
Performance and Indoor Thermal Comfort: A New Method Using Artificial Bee Colony (ABC). Energy and Buildings 131, 42-53.

Dogan, T., \& Reinhart, C. (2013). Automated Conversion of Architectural Massing Models into Thermal 'shoebox'models. Proceedings from BS2013: Building Simulation Conference, 3745-3752. Chambéry, France, 26-28 August 2013.

Dogan, T., C. Reinhart, and P. Michalatos (2016). Autozoner: An Algorithm for Automatic Thermal Zoning of Buildings with Unknown Interior Space Definitions. Journal of Building Performance Simulation 9 (2), 176-189.

Jones, N., McCrone, C., Walter, B., Pratt, K., \& Greenberg, D. (2013). Automated Translation and Thermal Zoning of Digital Building Models for Energy Analysis. Proceedings from BS2013: Building Simulation Conference, 202-209. Chambéry, France, 26-28 August 2013.

Krieg, O. D., T. Schwinn, A. Menges, J. Li, J. Knippers, A. Schmitt, and V. Schwieger (2015). Biomimetic Lightweight Timber Plate Shells: Computational Integration of Robotic Fabrication, Architectural Geometry and Structural Design. In Block P., Knippers J., Mitra N., Wang W. Advances in Architectural Geometry 2014, 109-125. Springer. Cham (Switzerland).

Picco, M., and M. Marengo (2015). On the Impact of Simplifications on Building Energy Simulation for Early Stage Building Design. Journal of Engineering and Architecture 3 (1): 66-78.

Rörig, T., S. Sechelmann, A. Kycia, and M. Fleischmann (2015). Surface Panelization Using Periodic Conformal Maps. In Block P., Knippers J., Mitra N., Wang W. Advances in Architectural Geometry 2014, 199-214. Springer. Cham (Switzerland).

Roudsari, M., Pak, M., \& Smith, A. (2013). Ladybug: A Parametric Environmental Plugin for Grasshopper to Help Designers Create an Environmentally-Conscious Design. Proceedings from BS2013: Building Simulation Conference, 3128-3135. Chambéry, France, 26-28 August 2013.

Santos, L., S. Schleicher, and L. Caldas (2017). Automation of CAD Models to BEM Models for Performance Based Goal-Oriented Design Methods. Building and Environment 112, 144-58. https://doi.org/10.1016/j.buildenv.2016.10.015.

Sechelmann, S., T. Rörig, and A. Bobenko (2013). Quasiisothermic Mesh Layout. In Hesselgren, L., S. Sharma, J. Wallner, N. Baldassini, P. Bompas, J. Raynaud. Advances in Architectural Geometry 2012, 243-58. Springer. Vienna (Austria). https://doi.org/10.1007/978-3-7091-1251-9_20. 